\title{
AMP-activated protein kinase mediates ischemic glucose uptake and prevents postischemic cardiac dysfunction, apoptosis, and injury
}

\author{
Raymond R. Russell III,1 Ji Li, ${ }^{1}$ David L. Coven, ${ }^{1}$ Marc Pypaert, ${ }^{2}$ Christoph Zechner, ${ }^{1}$ \\ Monica Palmeri, ${ }^{1}$ Frank J. Giordano,, ${ }^{1}$ James Mu, ${ }^{3}$ Morris J. Birnbaum, ${ }^{3}$ and Lawrence H. Young ${ }^{1}$ \\ 1Department of Internal Medicine (Section of Cardiovascular Medicine) and 2Department of Cell Biology, Yale University School of Medicine, \\ New Haven, Connecticut, USA. ${ }^{3}$ Howard Hughes Medical Institute, University of Pennsylvania, Philadelphia, Pennsylvania, USA.
}

\begin{abstract}
AMP-activated protein kinase (AMPK) is an important regulator of diverse cellular pathways in the setting of energetic stress. Whether AMPK plays a critical role in the metabolic and functional responses to myocardial ischemia and reperfusion remains uncertain. We examined the cardiac consequences of long-term inhibition of AMPK activity in transgenic mice expressing a kinase dead (KD) form of the enzyme. The KD mice had normal fractional shortening and no heart failure, cardiac hypertrophy, or fibrosis, although the in vivo left ventricular $(\mathrm{LV}) \mathrm{dP} / \mathrm{dt}$ was lower than that in WT hearts. During low-flow ischemia and postischemic reperfusion in vitro, KD hearts failed to augment glucose uptake and glycolysis, although glucose transporter content and insulin-stimulated glucose uptake were normal. KD hearts also failed to increase fatty acid oxidation during reperfusion. Furthermore, KD hearts demonstrated significantly impaired recovery of LV contractile function during postischemic reperfusion that was associated with a lower ATP content and increased injury compared with WT hearts. Caspase-3 activity and TUNEL-staining were increased in KD hearts after ischemia and reperfusion. Thus, AMPK is responsible for activation of glucose uptake and glycolysis during low-flow ischemia and plays an important protective role in limiting damage and apoptotic activity associated with ischemia and reperfusion in the heart.
\end{abstract}

\section{Introduction}

AMP-activated protein kinase (AMPK) is a serine-threonine kinase, which is emerging as an important regulator of diverse cellular pathways in the setting of energetic stress $(1,2)$. AMPK modulates the rapid activation of major energy-generating metabolic pathways, stimulating the oxidation of FFAs $(3,4)$ and enhancing glucose uptake and glycolysis (5-9). More chronic activation of AMPK regulates metabolic flux by increasing the expression of genes encoding key metabolic proteins (10-12). In addition, AMPK also modulates integrated cellular processes and is instrumental in mitochondrial biogenesis in skeletal muscle $(13,14)$.

In the ischemic heart, there is decreased oxidative metabolism of both FFAs and glucose due to the diminished oxygen supply, but increased glucose transport and glycolytic ATP production (15). We have previously hypothesized that AMPK may play a role in enhancing glucose uptake and glycolysis during ischemia (5). Activation of glucose transport in skeletal muscle is dependent on AMPK activation during hypoxia (16-18), and to some extent during contraction (18), although the downstream targets of AMPK in GLUT4 vesicular trafficking are unknown. In the heart, we have demonstrated that pharmacologic activation of AMPK leads to glucose transporter

Nonstandard abbreviations used: acetyl-CoA carboxylase (ACC); 5-aminoimidazole-4-carboxamide-1- $\beta$-4-ribofuranoside (AICAR); AMP-activated protein kinase (AMPK); carnitine palmitoyltransferase-1 (CPT-1); kinase dead (KD); left ventricular (LV); LV developed pressure (LVDP); 6-phosphofructo-2-kinase (PFK-2).

Conflict of interest: The authors have declared that no conflict of interest exists.

Citation for this article: J. Clin. Invest. 114:495-503 (2004).

doi:10.1172/JCI200419297. translocation and increased glucose uptake (5). AMPK is also known to phosphorylate and activate 6-phosphofructo-2-kinase (PFK-2), which leads to the production of fructose 2,6-bisphosphate, an activator of glycolysis (6). During reperfusion after ischemia, myocardial FFA oxidation is thought to be increased by AMPK phosphorylation of acetyl-CoA carboxylase (ACC), which inhibits the enzyme and decreases the production of malonyl-CoA, an inhibitor of carnitine palmitoyltransferase-1 (CPT-1) (3, 19); this in turn decreases glucose oxidation through the Randle cycle (20). AMPK likely also modulates the activity of additional cellular pathways in ischemic hearts and those that have undergone ischemia/reperfusion, but it remains uncertain as to whether the overall action of AMPK is beneficial or detrimental in this setting $(21,22)$.

The overall aim of this study was to directly assess the role of AMPK in the metabolic and functional response of the heart during low-flow ischemia and postischemic reperfusion. Hearts from transgenic mice expressing a kinase dead (KD) mutation of the $\alpha 2$ catalytic subunit of AMPK (18) were examined to investigate whether AMPK deficiency impairs glucose uptake and glycolysis during ischemia and alters FFA oxidation and glucose metabolism during reperfusion. In addition, we assessed the functional consequences of AMPK deficiency on left ventricular (LV) contractile function and on the degree of LV dysfunction, necrosis, and apoptotic activity following ischemia and reperfusion.

\section{Results}

Cardiac phenotype of KD mice. Hearts from the KD1 line of KD transgenic mice had sufficient expression of the c-myc-tagged K45R $\alpha 2$ 


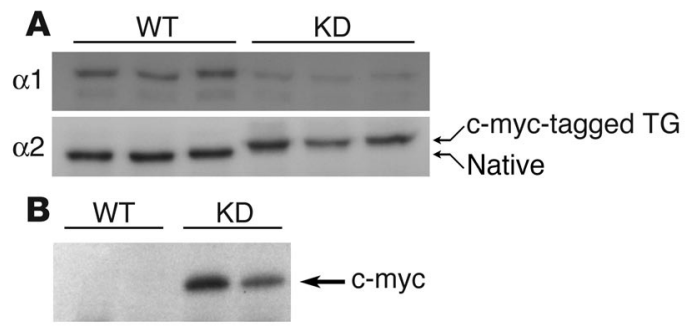

Figure 1

Protein expression of the native and mutant catalytic $\alpha$ subunits of AMPK in WT and AMPK KD hearts. Immunoblots showing the expression of native and mutant $\alpha$ isoforms of the catalytic subunit of AMPK in hearts from KD (K45R $\alpha 2$ mutation) and WT mice (A). Immunoblot confirming the expression of the c-myc-tagged transgene in KD hearts (B). Note that the KD transgenic $\alpha 2$ isoform containing the K45R mutation and c-myc tag migrates more slowly than the WT $\alpha 2$ isoform. TG, transgene project.

AMPK isoform to effectively replace native AMPK isoforms, as shown by immunoblotting heart extracts (Figure 1). The KD protein migrates slower than the WT $\alpha 2$ isoform on SDS-PAGE because of the c-myc tag. This reduction in the content of native $\alpha$ subunits is similar to findings in skeletal muscle, where expression of the K45R isoform effectively competes with native $\alpha$ isoforms for incorporation into the heterotrimeric AMPK complex and those $\alpha$ subunits that are not bound to $\beta$ and $\gamma$ subunits likely are degraded (18). Endothelial cells contain the $\alpha 1$ isoform (23), which may contribute to the $\alpha 1$ protein detected on immunoblots in these hearts with myocyte-specific expression of the KD protein.

Previous studies have demonstrated that hearts from transgenic mice with mutations in genes regulating metabolic pathways can have a number of morphologic abnormalities, including hypertrophy and fibrosis (24-27). The KD hearts were slightly smaller than those of their WT littermates, with approximately $10 \%$ reduction in the heart weight-to-body weight ratio and a trend toward reduced myocyte diameter (Table 1), but were otherwise morphologically normal. They had no evidence of cardiac fibrosis based on trichrome staining or gross ultrastructural abnormalities based on analysis of electron micrographs (Figure 2).

To examine KD mouse hearts with respect to in vivo LV function, echocardiographic and LV hemodynamic measurements were performed. While the LV end-systolic and end-diastolic dimensions and fractional shortening were similar and in a normal range in KD and WT littermate hearts, the echocardiographic fractional wall thickening was slightly lower in the KD hearts compared with WT hearts (Table 1). The in vivo LV diastolic and systolic pressures were similar in the two groups, but there was mildly decreased LV $+\mathrm{dP} / \mathrm{dt}$ and $-\mathrm{dP} / \mathrm{dt}$ in the KD hearts (Table 1 ).

Effects of ischemia and reperfusion in AMPK activity. AMPK activity was measured in $\alpha 1$ and $\alpha 2$ immunoprecipitates at baseline during low-flow ischemia and during reperfusion. Under baseline conditions, KD hearts had negligible $\alpha 2$ activity, but normal baseline $\alpha 1$ activity, despite their reduction in $\alpha 1$ protein (Figure 3 ). During low-flow ischemia, both $\alpha 1$ and $\alpha 2$ activity increased approximately threefold in the WT hearts, but neither increased significantly in the KD hearts. Taken together, these results indicate that there is complete inactivation of $\alpha 2$ activity, while the small amount of $\alpha 1$ that is present in the heart is maximally active at baseline and is not significantly activated during ischemia. Furthermore, during postischemic reperfusion, $\alpha 1$ and $\alpha 2$ activities remained elevated in the WT hearts, but were similar to baseline in the KD hearts.

Glucose uptake during ischemia and reperfusion. While pharmacologic activation of AMPK by 5 -aminoimidazole-4-carboxamide-1- $\beta-4$ ribofuranoside (AICAR) stimulates the translocation of GLUT4 and increases glucose uptake (5), definitive evidence that AMPK mediates the activation of heart glucose transport during ischemia is lacking. To address this issue, we assessed glucose uptake in WT and KD hearts perfused with glucose and fatty acids and subjected them to low-flow ischemia and reperfusion. In the WT hearts, glucose uptake increased twofold during ischemia and remained elevated during reperfusion (Figure 4A). Although glucose uptake under baseline conditions was normal in KD hearts, there was no increase in glucose uptake during ischemia or reperfusion, supporting a critical role for AMPK in mediating enhanced glucose transport during both ischemia and reperfusion. Increased glucose uptake during ischemia was associated with enhanced glycolysis, as evidenced by increased lactate production in the WT hearts (Figure 4B). In contrast, the failure to increase exogenous glucose uptake resulted in less of an increase in lactate release during ischemia in the KD hearts. While lactate production remained elevated during reperfusion in WT hearts, indicating persistently increased glycolysis, it returned to baseline values in KD hearts.

To exclude the possibility that KD hearts have a more generalized impairment in the activation of glucose uptake, we examined the content of glucose transporters and the ability of insulin to stimulate glucose uptake in these hearts. The total GLUT4 and GLUT1 contents were similar in KD and WT hearts, both at baseline and following ischemia/reperfusion (Figure 4D). When hearts were perfused with insulin $(1,000 \mu \mathrm{U} / \mathrm{ml})$, the glucose uptake increased

\section{Table 1}

Morphologic, hemodynamic, and echocardiographic characteristics of WT and AMPK KD mice
WT

Morphologic $(\boldsymbol{n}=\mathbf{1 6}[\mathrm{WT}] ; \boldsymbol{n}=\mathbf{1 9}$
BW $(\mathrm{g})$
HW $(\mathrm{mg})$
HW/BW $(\mathrm{mg} / \mathrm{g})$
Myocyte diameter $(\mu \mathrm{m})^{\mathrm{A}}$
Hemodynamic $(\boldsymbol{n}=\mathbf{5}$ for both)
LV peak systolic pressure $(\mathrm{mmHg})$
LV end-diastolic pressure $(\mathrm{mmHg})$
+dP/dt $\left(\mathrm{mmHg}_{\mathrm{mm}} \times \mathrm{sec}^{-1}\right)$
-dP/dt $\left(\mathrm{mmHg}^{-1} \mathrm{sec}^{-1}\right)$
Heart rate $\left(\mathrm{min}^{-1}\right)$

Echocardiographic ( $n=10$ for both)

Wall thickness $(\mathrm{mm})$

LV diastolic diameter ( $\mathrm{mm}$ )

LV systolic diameter $(\mathrm{mm})$

Fractional wall thickening (\%)

Fractional shortening (\%)

Heart rate $\left(\mathrm{min}^{-1}\right)$

$\begin{array}{cc}40.8 \pm 1.2 & 41.3 \pm 1.0 \\ 270 \pm 12 & 237 \pm 11^{\mathrm{B}} \\ 6.74 \pm 0.34 & 5.80 \pm 0.33^{\mathrm{B}} \\ 17.27 \pm 1.63 & 16.27 \pm 1.08\end{array}$

\begin{tabular}{|c|c|}
\hline $.9 \pm$ & $2.8 \pm$ \\
\hline $7.4 \pm 2.8$ & $6.1 \pm 0$. \\
\hline $65 \pm 1$ & 4 , \\
\hline+8 & 60 \\
\hline & \\
\hline
\end{tabular}

$1.07 \pm 0.01 \quad 1.02 \pm 0.01$

$2.51 \pm 0.01 \quad 2.83 \pm 0.01$

$0.94 \pm 0.01 \quad 1.18 \pm 0.01$

$65.9 \pm 1.3 \quad 60.7 \pm 1.5^{\mathrm{B}}$

$63.2 \pm 2.2 \quad 58.9 \pm 3.7$

$532 \pm 30 \quad 534 \pm 13$
A total of 96 myocytes were measured for WT and 139 for KD hearts. Values are mean \pm SEM. AMyocyte diameter was measured on lowpower electron micrographs ( $n=2$ for WT and $n=3$ for KD hearts). ${ }^{\mathrm{B}} P<0.05$. HW, heart weight; BW, body weight. 


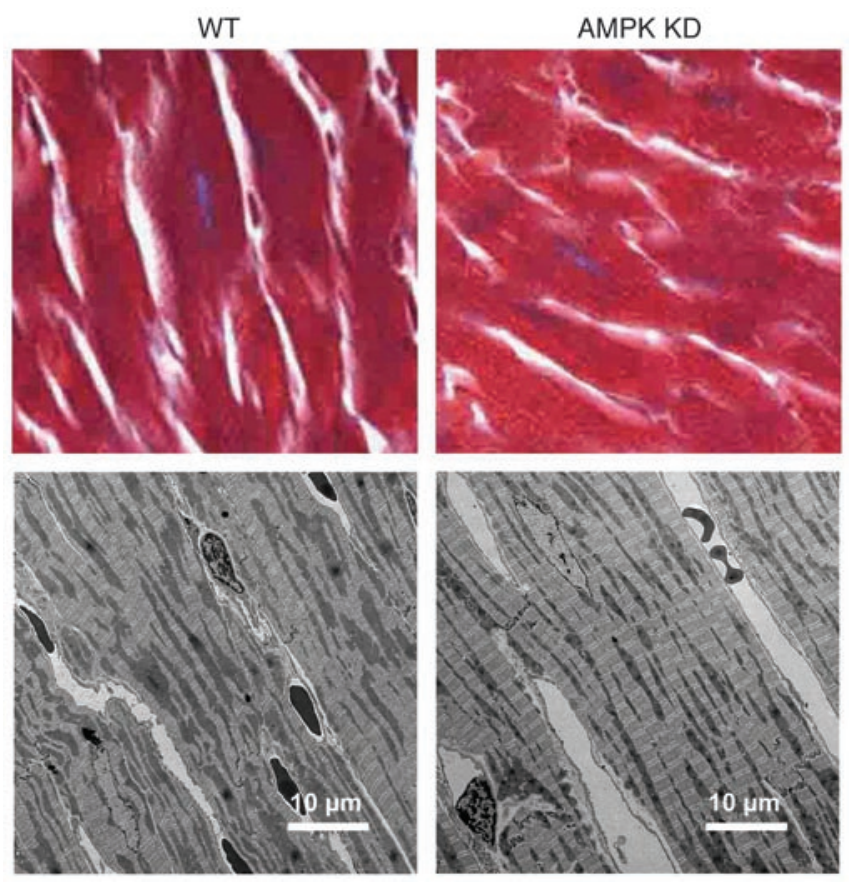

approximately threefold in both KD and WT hearts (Figure 4C). Thus, both baseline and insulin-stimulated glucose uptake were intact in the KD hearts, indicating that the impairment in glucose uptake was specific to the ischemia-activated AMPK pathway.

Fatty acid and glucose oxidation during ischemia and reperfusion. AMPK activation in the postischemic heart is associated with an increase in fatty acid oxidation by inhibiting ACC and decreasing the production of malonyl-CoA, which inhibits CPT-1 (3). To directly examine this issue, we evaluated the effect of loss of AMPK function on postischemic fatty acid and glucose oxidation. WT and KD hearts had similar rates of fatty acid oxidation at baseline, which fell with the decreased oxygen delivery associated with low-flow ischemia (Figure 5A). During reperfusion, however, fatty acid oxidation rates increased in the WT group compared with the baseline values. In contrast, in the KD group, fatty acid oxidation during reperfusion was lower than at baseline (Figure $5 \mathrm{~A}$ ), indicating the importance of AMPK in the augmentation of postischemic fatty acid oxidation. In addition, glucose oxidation rates were similar at baseline and decreased to a comparable level during low-flow ischemia in WT and KD hearts (Figure 5B). There was greater glucose oxidation during reperfusion than at baseline in the KD hearts, however, while there was no significant difference between glucose oxidation during baseline and reperfusion in the WT hearts (Figure 5B).

Effects of ischemia and reperfusion on myocardial glycogen and high-energy phosphate content. Hearts expressing mutations in the $\gamma$ regulatory subunit of AMPK have high AMPK activity and increased glycogen content (28). Interestingly, under baseline conditions, there was

\section{Figure 3}

$\alpha 2-$ and $\alpha 1$-specific AMPK activity from hearts from KD and WT mice after baseline perfusion period ( $n=3$ for both), after ischemia ( $n=4$ for both), and following 30 minutes of ischemia and 30 minutes of reperfusion ( $n=7$ for WT and $n=6$ for KD). ${ }^{\star} P<0.01$ versus baseline; $+P<0.01$ versus $W T$.

\section{Figure 2}

Light microscopy of trichrome-stained paraffin sections (upper panels: original magnification, $\times 40$ ) and electron micrographs (lower panels: original magnification:, $\times 1,200$ ) of hearts from WT (left panels) and AMPK KD (right panels) hearts. There is no evidence of increased fibrosis or ultrastructural changes in the KD hearts.

less glycogen in the KD compared with WT hearts (Table 2). The glycogen content decreased mildly after low-flow ischemia and by a similar amount (approximately $4 \mu \mathrm{mol} / \mathrm{g} / 30 \mathrm{~min}$ ) in $\mathrm{KD}$ and WT hearts. Glycogen returned to baseline values during reperfusion in both KD and WT hearts. These findings suggest that AMPK deficiency does not impair either the mobilization of glycogen during ischemia or its resynthesis during reperfusion.

The content of the high-energy phosphates, ATP and phosphocreatine, was also assessed following baseline perfusion, ischemia, and reperfusion (Table 2). Following baseline perfusion, there were similar levels of ATP and phosphocreatine in WT and KD hearts; there was also a similar decline in the contents of the high-energy phosphates at the end of ischemia. Following reperfusion, ATP content was lower in KD hearts, though it remained depressed in both WT and KD hearts. The phosphocreatine content after reperfusion also tended to be lower in KD hearts $(P<0.07)$, but returned toward baseline values in both WT and KD hearts.

$L V$ function during ischemia and reperfusion. The KD hearts also demonstrated significantly impaired recovery of postischemic LV contractile function in the setting of ischemia and reperfusion. When perfused in the Langendorff mode, there was a minor decrease in baseline LV contractility, as evidenced by a lower positive and negative $\mathrm{dP} / \mathrm{dt}$ and a trend toward reduced LV developed pressure (LVDP) in KD compared with WT littermate hearts (Figure 6). During low-flow ischemia, LV contractility decreased markedly in both WT and KD hearts, but the values for LVDP, $+\mathrm{dP} / \mathrm{dt}$, and $-\mathrm{dP} / \mathrm{dt}$ were significantly lower in the KD hearts. The recovery of $\mathrm{LV}$ function in the KD hearts was more dramatically impaired during postischemic reperfusion. Specifically, the LVDP, $+\mathrm{dP} / \mathrm{dt}$, and $-\mathrm{dP} / \mathrm{dt}$ were all significantly reduced, indicating diminished LV contractility and relaxation during reperfusion in KD compared with WT hearts (Figure 6). Interestingly, there were significant linear relationships between LVDP and ATP $(r=0.817$; $P<0.01)$ and phosphocreatine $(r=0.673 ; P<0.05)$ content during postischemic reperfusion.

Myocardial injury during ischemia and reperfusion. We next assessed whether the poor recovery of LV function was due to greater myocardial injury in KD than WT hearts. Ischemic injury is mediated in large part through the development of myocardial necrosis, which disrupts the sarcolemma and leads to the release
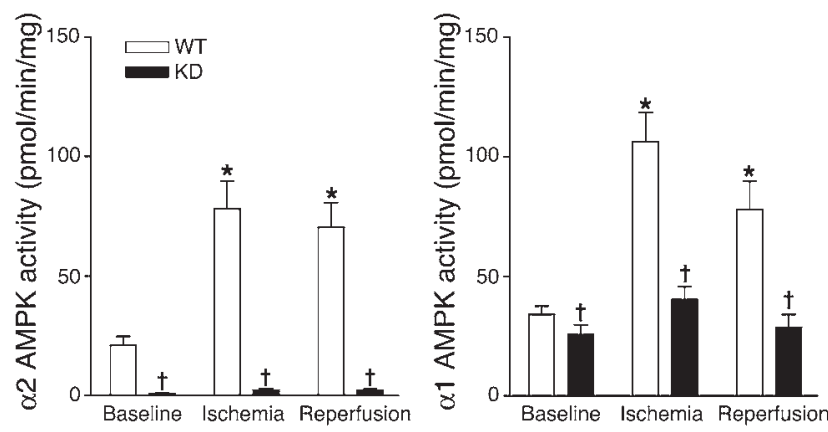

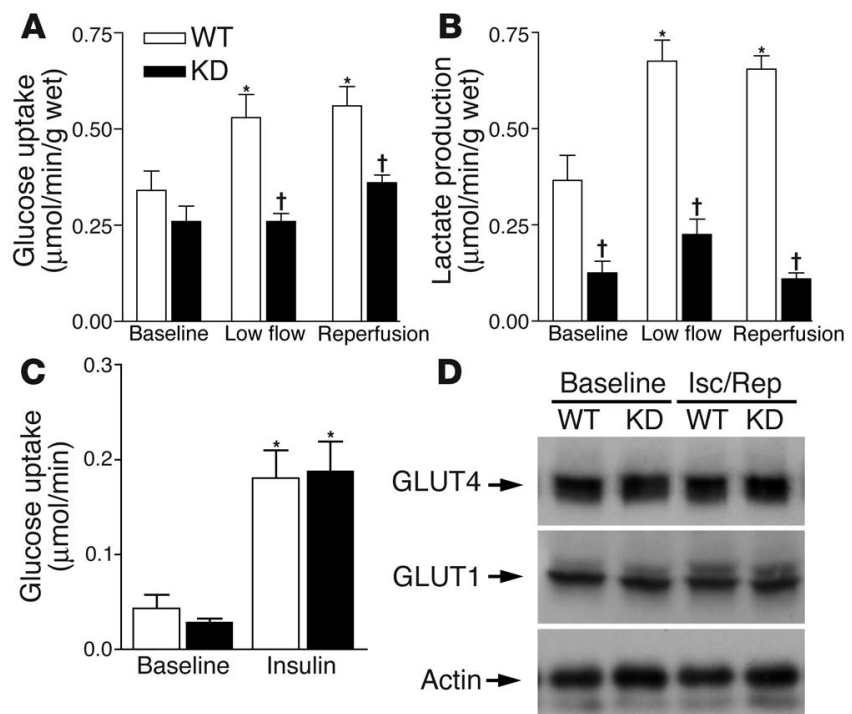

of intracellular enzymes. KD hearts had twofold greater release of creatine kinase and lactate dehydrogenase during both low-flow ischemia and postischemic reperfusion (Figure 7, A and B). These results indicate that the poor functional recovery of $\mathrm{KD}$ hearts is not simply a phenomenon of increased reperfusion injury, but rather is also a function of increased ischemic injury. Furthermore, because the hearts from KD animals were slightly smaller, the coronary flow during ischemia normalized to heart weight would tend to be higher; therefore, greater ischemic damage in KD hearts occurred despite a slightly less-severe ischemic insult compared with WT hearts.

Apoptotic activity after ischemia and reperfusion. Ischemia and reperfusion also initiate pathways leading to cardiac apoptosis (29). Baseline caspase- 3 activity was similar in KD and WT hearts. There was a significant increase in caspase-3 activity after ischemia/ reperfusion as compared with baseline values in the KD hearts (Figure 7C). In contrast, there was no increase in caspase-3 activity following ischemia/reperfusion in the WT hearts. In addition, confocal microscopy revealed a greater number of TUNEL-positive nuclei following ischemia/reperfusion in KD compared with WT hearts (Figure 7, D-F). As might be anticipated from the short reperfusion period (29), there was no evidence of DNA laddering on agarose gels after reperfusion in the KD hearts (data not shown). Therefore, taken together, these findings indicate that AMPK may play an important role in myocyte protection against ischemia/ reperfusion-mediated myocardial necrosis and apoptosis.

\section{Discussion}

These results demonstrate that AMPK has a critical role in mediating the metabolic and functional responses of the heart to low-flow ischemia and reperfusion. Specifically, loss of AMPK activity in the KD mouse heart is associated with an inability to augment glucose uptake and glycolysis as well as greater necrosis during low-flow ischemia. Furthermore, loss of AMPK activity has important functional consequences in the reperfused postischemic heart, including reduced recovery of LV contractile function, greater necrosis, and an increase in apoptotic activity. Taken together, these results indicate that AMPK has an important cardioprotective effect in the ischemic heart.

\section{Figure 4}

Glucose uptake, glycolysis, and GLUT expression in WT and KD hearts. Glucose uptake in hearts from KD transgenic $(n=6)$ and WT $(n=7)$ mice under baseline conditions, low-flow ischemia, and reperfusion (A). Lactate release from the same hearts (B). Stimulation of glucose uptake by insulin $(1,000 \mu \mathrm{U} / \mathrm{ml})$ in WT and KD hearts ( $n=3$ for both) (C) and immunoblot analysis of GLUT1 and GLUT4 expression in WT and KD hearts following baseline perfusion or ischemia/reperfusion (Isc/Rep) (D). ${ }^{*} P<0.05$ versus baseline; ${ }^{\dagger} P<0.05$ versus WT.

Previous results have demonstrated that low-flow ischemia increases myocardial glucose uptake by the translocation of glucose transporters from intracellular storage sites to the cell surface (15). Glucose uptake during ischemia is not mediated by the PI3K pathway, which is responsible for insulin-stimulated glucose uptake (5). The current observation that KD hearts failed to augment glucose uptake during ischemia provides strong evidence for the hypothesis that AMPK plays a critical role in stimulating glucose transport in the heart in response to metabolic and ischemic stress (5). Similarly, loss of AMPK activity inhibits hypoxiastimulated glucose uptake in skeletal muscle (18). Our results also complement recent observations on glucose transport during reperfusion after no-flow ischemia in a transgenic mouse that expresses an $\alpha 2$ D157A mutation in the heart (30). In that model, AMPK deficiency partially inhibited, but did not abolish, the stimulation of deoxyglucose uptake after 10 minutes of global no-flow ischemia. The greater inhibition of glucose uptake in the current experiments may be attributable to greater suppression of AMPK activity in this model, but it also raises the interesting possibility that additional mechanisms, which activate glucose transport, might be recruited during reperfusion following more severe no-flow ischemia. These two studies, taken together, make it extremely unlikely that impairment in ischemic glucose transport is due to nonspecific transgene insertion effects.

Although ischemia-activated glucose uptake was impaired in the KD hearts, insulin-stimulated glucose uptake was normal. This finding indicates that the KD hearts do not have a generalized impairment in the ability to activate glucose transport, implying that glucose transporters and the downstream mediators of GLUT vesicular trafficking are intact. While we found that GLUT4 and GLUT1 expression were normal in the KD hearts, the role of AMPK in modulating heart and skeletal muscle GLUT expression remains incompletely understood. In skeletal muscle, chronic AMPK activation with $\operatorname{AICAR}(10,11)$ or the expression of a constitutively active AMPK catalytic subunit
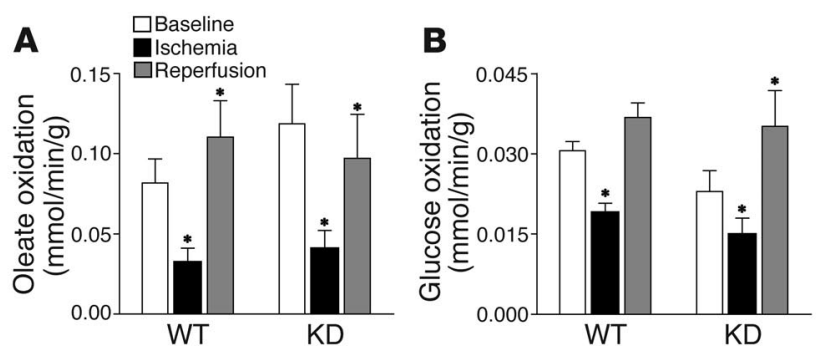

\section{Figure 5}

Rates of oleate oxidation (A) and glucose oxidation (B) in hearts from WT $(n=7)$ and KD transgenic $(n=6)$ mice under baseline conditions, low-flow ischemia, and reperfusion. ${ }^{*} P<0.05$ versus baseline. 


\section{Table 2}

Myocardial glycogen, ATP, and phosphocreatine content after ischemia and reperfusion in WT and AMPK KD hearts

\begin{tabular}{lcc}
\hline & WT & KD \\
Glycogen & & \\
Baseline & $12.5 \pm 1.4$ & $7.2 \pm 0.7^{\mathrm{A}}$ \\
Ischemia & $8.5 \pm 1.9$ & $3.3 \pm 0.9^{\mathrm{A}, \mathrm{B}}$ \\
Reperfusion & $12.6 \pm 2.1^{\mathrm{C}}$ & $6.1 \pm 0.5^{\mathrm{A}, \mathrm{C}}$ \\
ATP & & \\
Baseline & $4.2 \pm 0.4$ & $3.7 \pm 0.7$ \\
Ischemia & $1.9 \pm 0.3^{\mathrm{B}}$ & $1.3 \pm 0.1^{\mathrm{B}}$ \\
Reperfusion & $1.7 \pm 0.3^{\mathrm{B}}$ & $1.1 \pm 0.2^{\mathrm{AB}}$ \\
Phosphocreatine & & \\
Baseline & $5.1 \pm 0.7$ & $3.9 \pm 1.0$ \\
Ischemia & $2.2 \pm 0.8^{\mathrm{B}}$ & $1.0 \pm 0.4^{\mathrm{B}}$ \\
Reperfusion & $4.1 \pm 0.7$ & $2.6 \pm 0.6^{\mathrm{D}}$ \\
& & \\
\hline
\end{tabular}

Values ( $\mu \mathrm{mol} / \mathrm{g}$ wet weight) are reported as the mean \pm SEM of five to seven experiments. ${ }^{A} P<0.05$ versus $W T ;{ }^{B} P<0.05$ versus baseline; $C P<0.05$ versus ischemia; $D P<0.07$ versus WT.

(8) leads to the upregulation of GLUT4. However, similarly to our findings in the heart, AMPK deficiency in the KD mouse is not associated with decreased GLUT4 content in skeletal muscle (18). Thus, while increased AMPK might drive GLUT4 expression, AMPK activity does not appear to be required to maintain normal amounts of GLUT transporters in muscle.

This study also demonstrates decreased lactate production during both low-flow ischemia and reperfusion in KD hearts, which is in part the consequence of decreased glucose transport. While glucose transport across the sarcolemma is generally considered to be the flux-regulating step of glucose metabolism (31), there are other potential sites of regulation of glucose metabolism by AMPK. In particular, AMPK is also known to phosphorylate and activate PFK-2 during ischemia (6). Thus, an inability to activate PFK-2 during ischemia may have contributed to the decreased glycolytic flux and reduced lactate production observed in the KD hearts. Although the baseline glycogen concentration was reduced in KD hearts, the amount of glycogen consumed during ischemia was the same as that in WT hearts, suggesting that the diminished lactate production and greater injury was not due to impaired ischemic glycogen mobilization.

In addition to its role during ischemia, these studies demonstrate that AMPK has an important function in regulating myocardial metabolism during the postischemic reperfusion period. AMPK activity remained elevated in WT hearts during reperfusion, with little deactivation even 30 minutes after the reinstitution of normal perfusion. Prolonged elevation of AMPK activity was required for the persistently increased glucose uptake during reperfusion. In addition, AMPK is known to inhibit ACC, presumably leading to decreased malonyl-CoA, increased CPT-1 activity, and exaggerated FFA oxidation during reperfusion after ischemia $(3,19)$. Dur-

\section{Figure 6}

LVDP, heart rate $(\mathrm{HR}),+\mathrm{dP} / \mathrm{dt}$, and -dP/dt for WT $(n=7)$ and KD $(n=6)$ transgenic hearts before, during, and after low-flow ischemia. Horizontal lines identify time points with values that are significantly different $(P<0.05)$ from $t=0$. ${ }^{*} P<0.05$ versus WT. ing reperfusion, fatty acid oxidation rates increased above baseline values in WT hearts, but remained partially depressed in KD hearts, confirming the hypothesis that AMPK mediates increased fatty acid oxidation in the postischemic reperfused heart $(3,19)$.

Increased postischemic fatty acid oxidation is thought to inhibit glucose oxidation through Randle cycle mechanisms (20), resulting in the shunting of glycolytically derived pyruvate to lactate and effectively uncoupling glycolysis from glucose oxidation (32-34). In the reperfused WT hearts, we observed increases in fatty acid oxidation, glucose uptake, and lactate production, but no change in glucose oxidation compared with baseline. These results are consistent with the uncoupling of glycolysis from glucose oxidation, although a significant fall in glucose oxidation was not evident. In contrast, in the reperfused KD hearts, fatty acid oxidation was lower, glucose uptake and lactate production unchanged, and glucose oxidation was higher compared with baseline. These results suggest less uncoupling between glycolysis and glucose oxidation in the KD hearts, but also may reflect some degree of preferential utilization of exogenous glucose for glycogen synthesis during reperfusion. The replenishment of glycogen was similar in KD and WT hearts during reperfusion, despite the
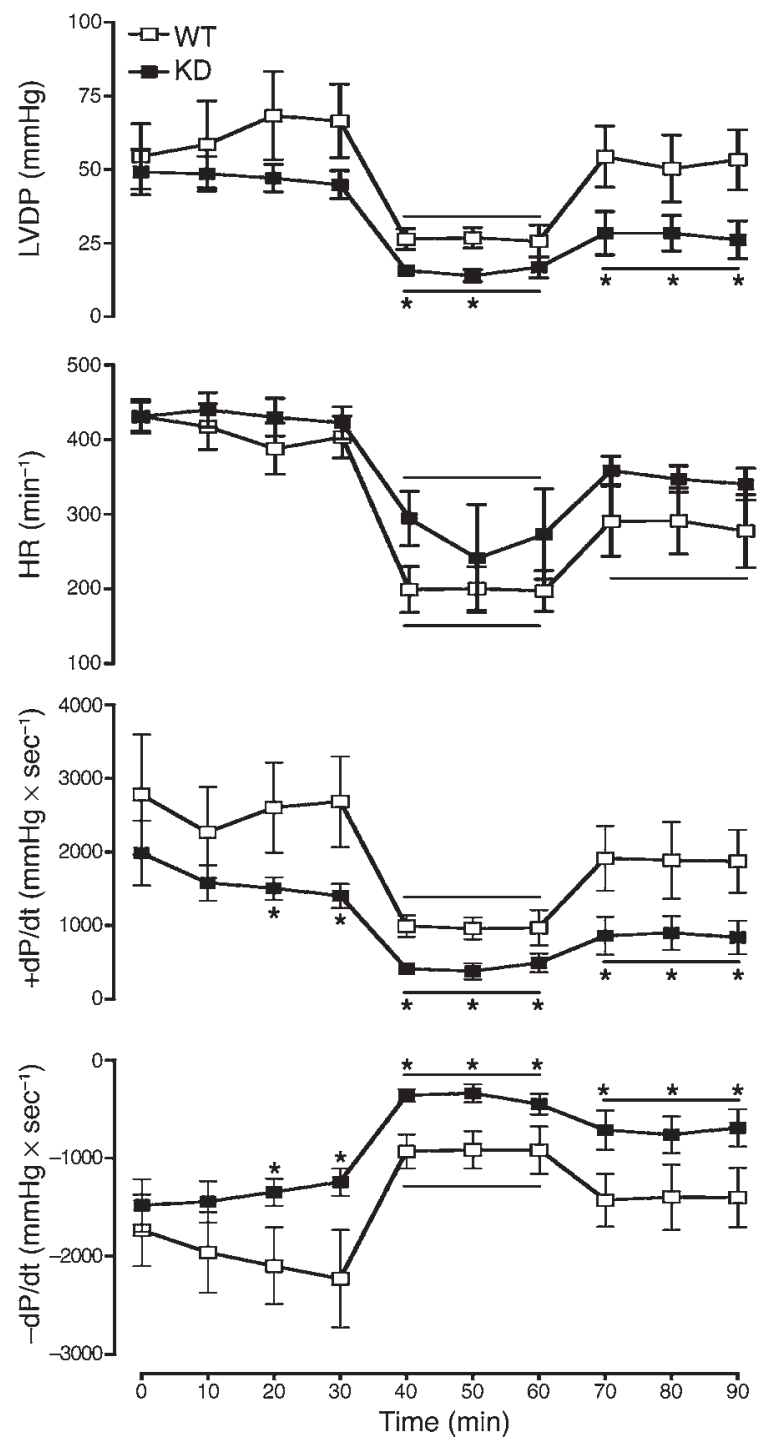

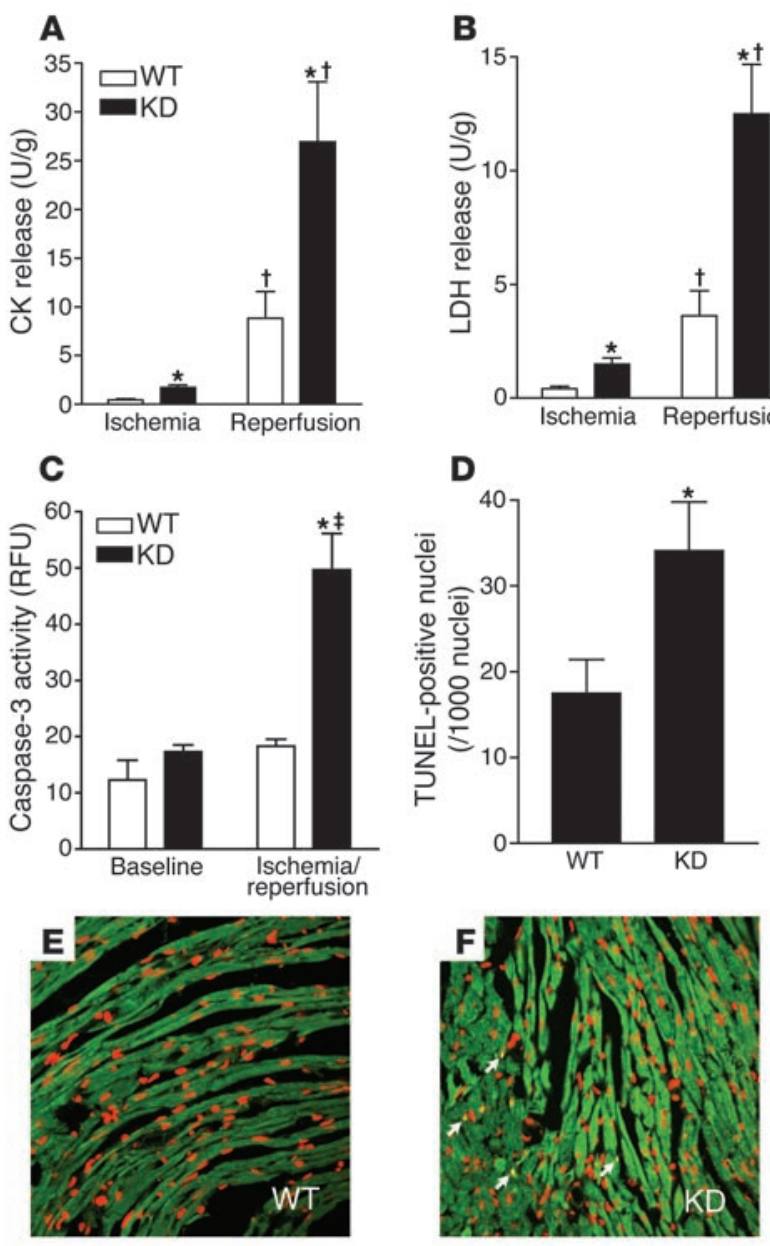

significantly lower rate of glucose uptake in KD hearts. However, glycogen replenishment was estimated from concentrations in different groups of mice, and studies designed to examine rates of glycogen synthesis are necessary to further characterize the fate of exogenous glucose in the reperfused KD hearts.

An important observation was that the KD hearts had a greater release of the intracellular enzymes lactate dehydrogenase and creatine kinase during both ischemia and reperfusion. These results indicate greater membrane damage and myocardial necrosis, suggesting that AMPK may activate downstream pathways that have cardioprotective roles during ischemia and reperfusion. In part, this cardioprotection may be related to AMPK-mediated increases in glucose uptake and glycolytic flux, which were impaired in KD hearts during low-flow ischemia. The importance of glucose uptake in the heart during low-flow ischemia is underscored by studies suggesting that GLUT4-deficient hearts also have impaired ischemia-stimulated glycolysis and mildly decreased postischemic contractile function (35). Similarly, inhibition of heart glucose uptake during ischemia by cytochalasin B results in poorer postischemic contractile function and greater myocyte damage (36).

Glucose uptake during ischemia and glycolytic ATP production may have a specific role in maintaining cell membrane integrity and ionic gradients during ischemia (37). Although there was not a significantly lower ATP content in KD hearts during ischemia, such measurements of total ATP in freeze-clamped hearts do not exclude the possibility that key subcellular pools

\section{Figure 7}

Myocardial injury and apoptotic activity following low-flow ischemia. Release of myocardial creatine kinase (CK) and lactate dehydrogenase $(\mathrm{LDH})$ from WT $(n=7)$ and $\mathrm{KD}(n=6)$ transgenic hearts during ischemia and reperfusion (A and $\mathbf{B}$ ). Caspase-3 activity following baseline perfusion ( $n=3$ for both groups) and following ischemia/reperfusion ( $n=7$ for WT and $n=6$ for KD) (C). Quantification of apoptotic nuclei from WT and KD hearts ( $n=3$ for both) following ischemia/reperfusion (D). Representative composite confocal photomicrographs of sections from WT and KD hearts following reperfusion ( $E$ and $F$ ). Hearts underwent TUNEL staining with fluorescein-labeled dUTP and counterstaining with propidium iodide. TUNEL-positive nuclei are stained yellow (arrows). ${ }^{*} P<0.05$ versus WT; ${ }^{\dagger} P<0.05$ versus ischemia; $¥ P<0.05$ versus baseline.

of ATP might be more compromised in the KD hearts. During reperfusion, the content of ATP remained depressed in both WT and KD hearts, but was lower in KD hearts. In addition, during reperfusion, the phosphocreatine content tended to be lower in KD hearts $(P=0.068)$. Taken together with the correlations between the high-energy phosphate content and LV function, these findings suggest that loss of the ability to activate AMPK may result in impaired energy metabolism that is associated with postischemic contractile dysfunction.

Because AMPK increases postischemic fatty acid oxidation, there has been concern that AMPK might contribute to myocardial reperfusion injury by uncoupling glycolysis and glucose oxidation and perpetuating intracellular acidosis (38). In this sense, AMPK deficiency might have been predicted to attenuate myocardial damage in the postischemic reperfused heart (21); however, despite the fact that KD hearts had blunted fatty acid oxidation and less lactate production during reperfusion, they demonstrated increased myocardial injury. These results are consistent with the contention that the metabolic effects of AMPK on glucose transport and glycolysis during low-flow ischemia are beneficial. It seems likely, however, that AMPK may activate additional pathways, which are cardioprotective during ischemia and reperfusion. Chronic lack of AMPK might also alter gene expression in KD hearts, making them more susceptible to ischemic injury. AMPK is also activated by $\mathrm{H}_{2} \mathrm{O}_{2}(39)$, and reactive oxygen species generation during reperfusion may account for the persistent AMPK activation. However, it is unknown whether AMPK modulates pathways involved in the generation and scavenging of reactive oxygen species. The effects of AMPK activation on mitochondrial permeability and function also require further investigation. It is also important to consider that the extent to which AMPK is cardioprotective may depend on the severity and duration of ischemia.

In addition to the greater degree of myocardial necrosis in KD hearts, there was an increase in caspase- 3 activity and a greater number of TUNEL-positive nuclei in cardiac myocytes in KD compared with WT hearts, suggesting greater activation of apoptotic pathways. Previous studies have demonstrated that increasing glucose uptake through overexpression of GLUT1 inhibits apoptosis in hypoxic cells $(40,41)$. The pathway by which AMPK may protect against apoptosis is not known, although increased glucose use also inhibits cytochrome $c$ release from the mitochondria (42). Recent studies in endothelial cells, thymocytes, and astrocytes have also demonstrated that activation of AMPK, either pharmacologically or by overexpression of a constitutively active form of the kinase, can decrease the rate of apoptosis induced by a variety of stimuli (43-45). Although 
AICAR has also been shown in some studies to stimulate apoptosis $(46,47)$, this may reflect a pharmacologic effect unrelated to AMPK activation (47).

While the current experiments suggest that AMPK deficiency has a detrimental effect on the heart during ischemia and reperfusion, whether or not enhanced activation of AMPK might be cardioprotective in this setting remains to be proven. It is interesting to note that a meta-analysis of clinical studies using AICAR in the setting of coronary artery bypass grafting demonstrated modest reductions in early cardiac death, myocardial infarction, and the need for LV assist device support (48). Historically, AICAR was developed as a drug (acadesine) designed to increase extracellular adenosine concentrations and adenosine receptor stimulation in ischemic tissue (49). Although not recognized at the time, it is possible that activation of AMPK may have played a role in these beneficial effects. With the ongoing development of newer agents, which are more specific and potent than AICAR, it will be of interest to further examine whether activation of heart AMPK is cardioprotective.

In this study, the KD hearts had lower baseline glycogen contents than WT hearts, consistent with recent observations of lower glycogen content in skeletal muscle from AMPK-deficient transgenic mice $(18,50)$. Although there is some evidence that AMPK inhibits, rather than activates, glycogen synthase (51), the exact role of AMPK in modulating glycogen metabolism remains uncertain. It is interesting that myocardial glycogen content is increased in both human and mouse hearts expressing what is thought to be a constitutively active mutant of the AMPK $\gamma$ subunit $(28,52,53)$. These hearts have marked accumulation of glycogen, to the extent that it produces pseudohypertrophy with increased ventricular wall thickness. In some cases, glycogen accumulation is also associated with ventricular pre-excitation characteristic of Wolf-Parkinson-White syndrome (29, $53,54)$. In this study we observed slightly smaller hearts in KD mice compared with their WT littermates. The changes in the glycogen content alone cannot explain these differences, however, and further work is needed to assess the effects of chronic AMPK deficiency on heart growth.

In summary, the present studies demonstrate that AMPK has an important role in regulating myocardial metabolism during lowflow ischemia/reperfusion and in limiting ischemic injury and apoptosis during postischemic reperfusion. Specifically, the loss of AMPK function results in impaired glucose uptake and glycolysis, poor recovery of postischemic function, and increased myocyte necrosis and apoptosis. It is unlikely that the beneficial effects of AMPK are limited strictly to metabolic effects during ischemia, and novel downstream targets of AMPK, which might be cardioprotective, need to be identified. While AMPK deficiency appears to be detrimental, further studies will be necessary to determine whether AMPK activators, as they become available, prevent myocardial injury during ischemia and reperfusion.

\section{Methods}

Muscle-specific $\alpha 2$ KD AMPK transgenic mouse. Transgenic mice (C57BL/6, $\mathrm{KD} 1$ line) that express a KD rat $\alpha 2$ isoform (K45R mutation), driven in heart and skeletal muscle by the muscle creatine kinase promoter, were studied (18). Male mice were studied at 4-6 months of age. No KD mice developed heart failure when studied out to 1 year. WT littermates served as the control group for comparison in all experiments. All studies were approved by the Yale Animal Care and Use Committee.
Cardiac phenotype. Heart weight, including ventricles and atria, was measured and expressed as a percentage of body weight. Sections of KD and WT hearts were fixed with $2 \%$ paraformaldehyde for microscopy (5) and stained with H\&E for histology and with trichrome stain to assess the degree of extracellular fibrosis. Heart tissue was also fixed in $2.5 \%$ glutaraldehyde by perfusion, processed, and sectioned for electron microscopy as previously described (14) to examine cardiac morphology, myocyte diameter, and ultrastructure. LV function was assessed echocardiographically with a $15-\mathrm{MHz}$ probe (Philips Medical Systems, Andover, Massachusetts, USA) after inhalation anesthesia (0.5-1\% isoflurane). LV cavity dimensions, anterior and posterior wall thicknesses, end-systolic fractional shortening, and percentage of wall thickening were measured from M-mode echocardiograms $(55,56)$. In addition, direct $\mathrm{LV}$ hemodynamic measurements (LV pressure, maximal $+\mathrm{dP} / \mathrm{dt}$, minimal $-\mathrm{dP} / \mathrm{dt}$ ) were obtained using a $1.4 \mathrm{~F}$ micromanometer-tipped pressure transducer catheter (Millar Instruments Inc., Houston, Texas, USA) introduced into the left ventricle through the right carotid artery after anesthesia with xylazine $(5 \mathrm{mg} / \mathrm{kg})$ and ketamine $(100 \mathrm{mg} / \mathrm{kg})(57)$.

Mouse heart perfusions. Isolated mouse hearts were retrogradely perfused with Krebs-Henseleit buffer containing $7 \mathrm{mM}$ glucose, $0.4 \mathrm{mM}$ oleate, $1 \% \mathrm{BSA}$, and a low fasting concentration of insulin $(10 \mu \mathrm{U} / \mathrm{ml})$. Initial experiments were performed to determine the rate of glucose transport and glycolysis at baseline, during low-flow ischemia, and during postischemic reperfusion. Hearts were initially perfused at a constant aortic pressure of $80 \mathrm{cmH}_{2} \mathrm{O}$ at baseline for 30 minutes, flow was then reduced to approximately $20-25 \%$ of baseline for 30 minutes of lowflow ischemic perfusion, and then hearts were reperfused at a constant aortic pressure of $80 \mathrm{cmH}_{2} \mathrm{O}$ for an additional 30 minutes. To determine whether the expression of the KD form of AMPK affects insulin stimulation of glucose transport, additional perfusions were performed in which the insulin concentration was increased to $1,000 \mu \mathrm{U} / \mathrm{ml}$ for 30 minutes after baseline perfusion for 30 minutes as described above. Glucose uptake was determined as described below.

A second series of experiments was performed to assess the effects of low-flow ischemia and reperfusion on heart glucose and fatty acid oxidation, LV function, and myocardial injury. In these experiments, constant flow perfusion was achieved using a peristaltic pump to produce lowflow ischemia with approximately $80 \%$ flow reduction. Following initial perfusion at $80 \mathrm{mmHg}$ for approximately 5 minutes to flush out residual blood from the heart and allow stabilization, hearts were perfused at a baseline flow of $4 \mathrm{ml} / \mathrm{min}$ (30 minutes), ischemia at $0.75 \mathrm{ml} / \mathrm{min}$ (30 minutes), and reperfusion at $4 \mathrm{ml} / \mathrm{min}$ (30 minutes). These perfusions were performed with radiolabeled glucose and oleate as described below. A fluid-filled latex balloon connected to a solid-state pressure transducer (Millar Instruments Inc.) was inserted into the left ventricle through a left atriotomy to measure pressure. LVDP, the first derivative of LVDP $(\mathrm{dP} / \mathrm{dt})$, and heart rate were recorded using a digital acquisition system (ADInstruments, Colorado Springs, Colorado, USA) at a balloon volume that resulted in a baseline LV end-diastolic pressure of $5 \mathrm{mmHg}$. Hearts were freeze-clamped after either the baseline, ischemia, or reperfusion periods and stored at $-80^{\circ} \mathrm{C}$ until the time of assay.

Metabolic analysis. Rates of glucose transport and phosphorylation were determined from the production of tritiated water from $\left[2-{ }^{3} \mathrm{H}\right]$ glucose (58). Lactate production was determined by measuring the perfusate lactate concentration using an automated analyzer (Stat 2300; Yellow Springs Instrument Co., Yellow Springs, Ohio, USA). The rate of glucose oxidation was determined by the production of ${ }^{14} \mathrm{CO}_{2}$ from $\left[\mathrm{U}-{ }^{14} \mathrm{C}\right]$ glucose, and the rate of fatty acid oxidation was determined by the production of ${ }^{3} \mathrm{H}_{2} \mathrm{O}$ from $\left[9,10-{ }^{3} \mathrm{H}\right]$ oleate (59). Glycogen content was determined from methanol precipitates of $\mathrm{KOH}$-digested tissue using 
the amyloglucosidase method (60). ATP and phosphocreatine were determined spectrophotometrically from neutralized perchloric acid extracts of tissue samples (61).

Analysis of AMPK expression and activity and GLUT4 expression. Immunoblot analysis of proteins separated by SDS-PAGE and electrophoretically transferred to PVDF membranes was performed to determine the expression of the native $\alpha 1$ and $\alpha 2$ subunits of AMPK and the KD $\alpha 2$ protein. Polyclonal sheep Ab's to $\alpha 1$ and $\alpha 2$ were generously provided by D. Grahame Hardie (University of Dundee, Dundee, United Kingdom) (62). A mAb to the c-myc epitope (Santa Cruz Biotechnology Inc., Santa Cruz, California, USA) in the KD $\alpha 2$ protein was used to further confirm transgene expression. Isoform-specific ( $\alpha 1$ and $\alpha 2)$ AMPK activity was determined using a previously described immune complex kinase assay (62). GLUT4 and GLUT1 expression were determined by immunoblot analysis of crude membrane fractions from heart homogenates using polyclonal Ab's kindly provided by Samuel Cushman (NIH, National Institute of Diabetes and Digestive and Kidney Diseases, Bethesda, Maryland, USA) (15).

Analysis of myocyte necrosis and apoptosis. Myocyte necrosis was assessed by determining the release of creatine kinase (Catachem Inc., Bridgeport, Connecticut, USA) and lactate dehydrogenase (Sigma-Aldrich, St. Louis, Missouri, USA) into the perfusate using commercially available spectrophotometric assays. The degree of apoptotic activity was assessed by measuring caspase- 3 activity in tissue homogenates using a commercially available fluorimetric assay (Oncogene Research Products, San Diego, California, USA). In addition, TUNEL staining using fluorescein-labeled dUTP (Roche Diagnostics, Indianapolis, Indiana, USA) and counterstaining with $0.5 \mu \mathrm{g} / \mathrm{ml}$ propidium iodide was performed on 5 - $\mu \mathrm{m}$ sections from hearts fixed by paraformaldehyde perfusion and processed as previously described (5). Slides were examined in a blinded fashion for apoptotic nuclei using a Zeiss LSM410 confocal microscope (Carl Zeiss Inc., Thornwood, New York, USA).
Statistical analysis. Values are reported as the mean plus or minus SEM. Differences between WT and KD animals were assessed by a Student $t$ test. Changes in metabolic parameters and LV function during ischemia and reperfusion between WT and KD transgenic animals were determined by ANOVA with repeated measures using Tukey's test for post hoc comparisons. A $P$ value of less than 0.05 was considered statistically significant.

\section{Acknowledgments}

The authors wish to express their appreciation to Syed Hasan, Carolyn Marks, Yan Huang, Lin Cong, and Jennifer Hu for their expert assistance with these experiments. This work was supported by United States Public Health Service grants RO1 HL63811 (to L.H. Young) and U24 DK59635 (to R.R. Russell) and National Research Service Award HL10301 (to D.L. Coven). C. Zechner was the recipient of a student fellowship from the German Academic Exchange Service (DAAD). M.J. Birnbaum is an Investigator of the Howard Hughes Medical Institute.

Received for publication June 24, 2003, and accepted in revised form June 8, 2004.

Address correspondence to: Lawrence H. Young, Fitkin 3, 333 Cedar Street, New Haven, Connecticut 06520, USA. Phone: (203) 785-4102; Fax: (203) 785-7567; E-mail: lawrence.young@yale.edu.

This work was presented in part at the Second International AMPK Symposium in Dundee, United Kingdom, on September 12-14, 2002, and the Scientific Sessions 2002 of the American Heart Association, Chicago, Illinois, USA, on November 17-20, 2002.
1. Hardie, D., Scott, J., Pan, D., and Hudson, E. 2003. Management of cellular energy by the AMP-activated protein kinase system. FEBS Lett. 546:113-120.

2. Kemp, B.E., et al. 2003. AMP-activated protein kinase, super metabolic regulator. Biochem Soc. Trans. 31:162-168.

3. Kudo, N., et al. 1996. Characterization of 5'AMPactivated protein kinase activity in the heart and its role in inhibiting acetyl-CoA carboxylase during reperfusion following ischemia. Biochim. Biophys. Acta. 1301:67-75.

4. Winder, W., and Hardie, D. 1996. Inactivation of acetyl-CoA carboxylase and activation of AMP-activated protein kinase in muscle during exercise. Am J. Physiol. 270:E299-E304.

5. Russell, R., Bergeron, R., Shulman, G., and Young, L. 1999. Translocation of myocardial GLUT4 and increased glucose uptake through activation of AMP-activated protein kinase by AICAR. Am. J Physiol. 277:H643-H649.

6. Marsin, A.S., et al. 2000. Phosphorylation and activation of heart PFK-2 by AMPK has a role in the stimulation of glycolysis during ischaemia. Curr Biol. 10:1247-1255.

7. Merrill, G., Kurth, E., Hardie, D., and Winder, W. 1997. AICA riboside increases AMP-activated protein kinase, fatty acid oxidation, and glucose uptake in rat muscle. Am. J. Physiol. 273:E1107-E1112.

8. Fryer, L.G., et al. 2002. Characterization of the role of the AMP-activated protein kinase in the stimulation of glucose transport in skeletal muscle cells Biochem. J. 363:167-174.

9. Kurth-Kraczek, E., Hirshman, M., Goodyear, L., and Winder, W. 1999. 5' AMP-activated protein kinase activation causes GLUT4 translocation in skeletal muscle. Diabetes. 48:1667-1671.

10. Ojuka, E.O., Nolte, L.A., and Holloszy, J.O. 2000 Increased expression of GLUT-4 and hexokinase in rat epitrochlearis muscles exposed to AICAR in vitro. J. Appl. Physiol. 88:1072-1075.

11. Holmes, B.F., Kurth-Kraczek, E.J., and Winder, W.W. 1999. Chronic activation of 5'-AMP-activated protein kinase increases GLUT-4, hexokinase, and glycogen in muscle. J. Appl. Physiol. 87:1990-1995.

12. Zhou, M., Lin, B.Z., Coughlin, S., Vallega, G., and Pilch, P.F. 2000. UCP-3 expression in skeletal muscle: effects of exercise, hypoxia, and AMP-activated protein kinase. Am. J. Physiol. 279:E622-E629.

13. Bergeron, R., et al. 2001. Chronic activation of AMP kinase results in NRF-1 activation and mitochondrial biogenesis. Am. J. Physiol. 281:E1340-E1346.

14. Zong, H., et al. 2002. AMP kinase is required for mitochondrial biogenesis in skeletal muscle in response to chronic energy deprivation. Proc. Natl. Acad. Sci. U. S. A. 99:15983-15987.

15. Young, L., et al. 1997. Low flow ischemia leads to translocation of canine heart GLUT-4 and GLUT1 glucose transporters to the sarcolemma in vivo. Circulation. 95:415-422.

16. Hayashi, T., Hirshman, M.F., Kurth, E.J., Winder, W.W., and Goodyear, L.J. 1998. Evidence for 5' AMP-activated protein kinase mediation of the effect of muscle contraction on glucose transport. Diabetes. 47:1369-1373.

17. Hayashi, T., et al. 2000. Metabolic stress and altered glucose transport: activation of AMP-activated protein kinase as a unifying coupling mechanism. Diabetes. 49:527-531.

18. Mu, J., Brozinick, J.T., Jr., Valladares, O., Bucan, M., and Birnbaum, M.J. 2001. A role for AMP-activated protein kinase in contraction- and hypoxia-regulated glucose transport in skeletal muscle. Mol. Cell. 7:1085-1094

19. Kudo, N., Barr, A., Barr, R., Desai, S., and Lopaschuk, G. 1995. High rates of fatty acid oxi- dation during reperfusion of ischemic hearts are associated with a decrease in malonyl-CoA levels due to an increase in $5^{\prime}$-AMP-activated protein kinase inhibition of acetyl-CoA carboxylase. J. Biol. Chem. 270:17513-17520.

20. Randle, P., Newsholme, E., and Garland, P. 1964. Regulation of glucose uptake by muscle. 8. Effects of fatty acids, ketone bodies and pyruvate and of alloxan-diabetes and starvation on the uptake and metabolic rate of glucose in rat heart and diaphragm muscles. Biochem. J. 93:652-665.

21. Sambandam, N., and Lopaschuk, G.D. 2003. AMPactivated protein kinase (AMPK) control of fatty acid and glucose metabolism in the ischemic heart. Prog. Lipid Res. 42:238-256.

22. Hopkins, T.A., Dyck, J.R., and Lopaschuk, G.D. 2003. AMP-activated protein kinase regulation of fatty acid oxidation in the ischaemic heart. Biochem. Soc. Trans. 31:207-212.

23. Morrow, V.A., et al. 2003. Direct activation of AMPactivated protein kinase stimulates nitric-oxide synthesis in human aortic endothelial cells. J. Biol. Chem. 278:31629-31639.

24. Katz, E.B., Stenbit, A.E., Hatton, K., DePinho, R., and Charron, M.J. 1995. Cardiac and adipose tissue abnormalities but not diabetes in mice deficient in GLUT4. Nature. 377:151-155.

25. Zisman, A., et al. 2000. Targeted disruption of the glucose transporter 4 selectively in muscle causes insulin resistance and glucose intolerance. Nat. Med. 6:924-928.

26. Shioi, T., et al. 2000. The conserved phosphoinositide 3-kinase pathway determines heart size in mice. EMBO J. 19:2537-2548.

27. Watanabe, K., et al. 2000. Constitutive regulation of cardiac fatty acid metabolism through peroxisome proliferator-activated receptor alpha associated with age-dependent cardiac toxicity. J. Biol. 
Chem. 275:22293-22299.

28. Arad, M., et al. 2003. Transgenic mice overexpressing mutant PRKAG2 define the cause of Wolff-Parkinson-White syndrome in glycogen storage cardiomyopathy. Circulation. 107:2850-2856.

29. Krijnen, P.A.J., et al. 2002. Apoptosis in myocardial ischaemia and infarction. J. Clin. Pathol. 55:801-811.

30. Xing, Y., et al. 2003. Glucose metabolism and energy homeostasis in mouse hearts overexpressing dominant negative $\alpha 2$ subunit of AMP-activated protein kinase. J. Biol. Chem. 278:28372-28377.

31. Morgan, H., Cadenas, E., Regen, D., and Park, C. 1961. Regulation of glucose uptake in muscle. II. Rate-limiting steps and effects of insulin and anoxia in heart muscle from diabetic rats. J. Biol. Chem 236:262-268.

32. McVeigh, J., and Lopaschuk, G. 1990. Dichloroacetate stimulation of glucose oxidation improves recovery of perfused working heart. Am. J. Physiol. 259:H1070-H1085.

33. Lopaschuk, G.D., Spafford, M.A., Davies, N.J., and Wall, S.R. 1990. Glucose and palmitate oxidation in isolated working rat hearts reperfused after a period of transient global ischemia. Circ. Res. 66:546-553.

34. Lopaschuk, G., and Saddik, M. 1992. The relative contribution of glucose and fatty acids to ATP production in hearts reperfused following ischemia. Mol. Cell. Biochem. 116:111-116.

35. Tian, R., and Abel, E.D. 2001. Responses of GLUT4deficient hearts to ischemia underscore the importance of glycolysis. Circulation. 103:2961-2966.

36. Ramasamy, R., Hwang, Y.C., Whang, J., and Bergmann, S.R. 2001. Protection of ischemic hearts by high glucose is mediated, in part, by GLUT-4. Am. J. Physiol. 281:H290-H297.

37. King, L.M., and Opie, L.H. 1998. Glucose and glycogen utilisation in myocardial ischemia-changes in metabolism and consequences for the myocyte. Mol. Cell. Biochem. 180:3-26.

38. McCormack, J., Barr, R., Wolff, A., and Lopaschuk, G. 1996. Ranolazine stimulates glucose oxidation in normoxic, ischemic, and reperfused ischemic rat hearts. Circulation. 93:135-142.

39. Choi, S.L., et al. 2001. The regulation of AMP-activated protein kinase by $\mathrm{H}_{2} \mathrm{O}_{2}$. Biochem. Biophys. Res. Commun. 287:92-97.

40. Malhotra, R., and Brosius, F. 1999. Glucose uptake and glycolysis reduce hypoxia-induced apoptosis in cultured neonatal rat cardiac myocytes. J. Biol. Chem. 274:12567-12575.

41. Loberg, R.D., Vesely, E., and Brosius, F.C., III. 2002. Enhanced glycogen synthase kinase- $3 \beta$ activity mediates hypoxia-induced apoptosis of vascular smooth muscle cells and is prevented by glucose transport and metabolism. J. Biol. Chem. 277:41667-41673.

42. Malhotra, R., Lin, Z., Vincenz, C., and Brosius, F.C. III. 2001. Hypoxia induces apoptosis via two independent pathways in Jurkat cells: differential regulation by glucose. Am. J. Physiol. 281:C1596-C1603.

43. Blazquez, C., Geelen, M.J., Velasco, G., and Guzman, M. 2001. The AMP-activated protein kinase prevents ceramide synthesis de novo and apoptosis in astrocytes. FEBS Lett. 489:149-153.

44. Stefanelli, C., et al. 1998. Inhibition of glucocorticoid-induced apoptosis with 5 -aminoimidazole4-carboxamide ribonucleoside, a cell-permeable activator of AMP-activated protein kinase. Biochem. Biophys. Res. Commun. 243:821-826.

45. Ido, Y., Carling, D., and Ruderman, N. 2002. Hyperglycemia-induced apoptosis in human umbilical vein endothelial cells: inhibition by the AMP-activated protein kinase activation. Diabetes. 51:159-167.

46. Meisse, D., et al. 2002. Sustained activation of AMP-activated protein kinase induces c-Jun N-terminal kinase activation and apoptosis in liver cells. FEBS Lett. 526:38-42.

47. Lopez, J.M., Santidrian, A.F., Campas, C., and Gil, J. 2003. 5-Aminoimidazole-4-carboxamide riboside induces apoptosis in Jurkat cells, but the AMPactivated protein kinase is not involved. Biochem. J. 370:1027-1032.

48. Mangano, D.T. 1997. Effects of acadesine on myocardial infarction, stroke, and death following surgery. A meta-analysis of the 5 international randomized trials. The Multicenter Study of Perioperative Ischemia (McSPI) Research Group. JAMA. 277:325-332.

49. Zhao, Z.Q., et al. 1995. Acadesine reduces myocardial infarct size by an adenosine mediated mechanism. Cardiovasc. Res. 29:495-505.

50. Jorgensen, S.B., et al. 2004. Knockout of the alpha2 but not alpha1 5'-AMP-activated protein kinase isoform abolishes 5-aminoimidazole-4-carboxamide-1-beta-4-ribofuranosidebut not contrac- tion-induced glucose uptake in skeletal muscle. J. Biol. Chem. 279:1070-1079.

51. Wojtaszewski, J.F., Jorgensen, S.B., Hellsten, Y., Hardie, D.G., and Richter, E.A. 2002. Glycogendependent effects of 5-aminoimidazole-4-carboxamide (AICA)-riboside on AMP-activated protein kinase and glycogen synthase activities in rat skeletal muscle. Diabetes. 51:284-292.

52. Blair, E., et al. 2001. Mutations in the $\gamma 2$ subunit of AMP-activated protein kinase cause familial hypertrophic cardiomyopathy: evidence for the central role of energy compromise in disease pathogenesis. Hum. Mol. Genet. 10:1215-1220.

53. Arad, M., et al. 2002. Constitutively active AMP kinase mutations cause glycogen storage disease mimicking hypertrophic cardiomyopathy. J. Clin. Invest. 109:357-362. doi:10.1172/JCI200214571.

54. Gollob, M.H., et al. 2001. Novel PRKAG2 mutation responsible for the genetic syndrome of ventricular preexcitation and conduction system disease with childhood onset and absence of cardiac hypertrophy. Circulation. 104:3030-3033.

55. Gardin, J.M., Siri, F.M., Kitsis, R.N., Edwards, J.G., and Leinwand, L.A. 1995. Echocardiographic assessment of left ventricular mass and systolic function in mice. Circ. Res. 76:907-914.

56. Tanaka, N., et al. 1996. Transthoracic echocardiography in models of cardiac disease in the mouse. Circulation. 94:1109-1117.

57. Huang, Y., et al. 2004. Cardiac myocyte-specific HIF- $1 \alpha$ deletion alters vascularization, energy availability, calcium flux and contractility in the normoxic heart. FASEB J. 18:1138-1140.

58. Katz, J., and Dunn, A. 1967. Glucose-2-t as a tracer for glucose metabolism. Biochemistry. 6:1-5.

59. Lopaschuk, G.D., and Barr, R.L. 1997. Measurements of fatty acid and carbohydrate metabolism in the isolated working rat heart. Mol. Cell. Biochem. 172:137-147.

60. Russell, R., Nguyen, V., Mrus, J., and Taegtmeyer, H. 1992. Fasting and lactate unmask insulin responsiveness in the isolated working rat heart. Am. J. Physiol. 263:E556-E561.

61. Bergmeyer, H. 1974. Methods of enzymatic analysis. 3rd edition. Verlag-Chemie, Weinheim/Academic Press Inc. New York, New York, USA. 2000 pp.

62. Coven, D.L., et al. 2003. Physiological role of AMPactivated protein kinase in the heart: graded activation during exercise. Am. J. Physiol. 285:E629-E636. 